\title{
The influence of hydrogenation on superconducting properties of $\mathrm{MgB}_{2}$
}

\author{
A. J. Zaleski, W. Iwasieczko, M. Tkacz ${ }^{1}$, D. Kaczorowski, H. Drulis, \\ O. J. Zogat ${ }^{1}$, and J. Klamut ${ }^{2}$ \\ Institute of Low Temperature and Structure Research, PAS, Wroclaw, Poland \\ ${ }^{1}$ Institute of Physical Chemistry, PAS, Warsaw, Poland \\ ${ }^{2}$ International Laboratory of High Magnetic Fields and Low Temperatures, Wroclaw, Poland \\ E-mail: zaleski@int.pan.wroc.pl \\ Received June 5, 2001
}

\begin{abstract}
In the paper we present the results of the ac susceptibility measurements of two newly discovered superconducting diboride $-\mathrm{MgB}_{2}$.

PACS: 74.25.Ha, 74.72.-h
\end{abstract}

\section{Introduction}

At the very beginning of new millennium Akimitsu proved [1] that it is still possible to discover superconductivity, with rather high critical temperature, for the compound which comes from already well investigated family of diborides, and was commercially available. Since this discovery $\mathrm{MgB}_{2}$, with its critical temperature $T_{c}=38 \mathrm{~K}$, had been investigated with unprecedented speed. After few months almost every characteristic of the material was determined.

First information about the superconducting properties of the compounds with $\mathrm{AlB}_{2}$-type structure were published already at 1970 by Cooper et al. [2]. They found that hexagonal $\mathrm{NbB}_{2}$ or $\mathrm{MoB}_{2}$ as cast are not superconducting, but increasing the amount of boron to about 2.5 or substituting with Sc, Y, Zr, Mo, Ru, Hf or Th for Nb or additionally for $\mathrm{Al}, \mathrm{Ti}, \mathrm{V}, \mathrm{Ta}$ or $\mathrm{Au}$ for Mo resulted with superconducting material with critical temperature peaking with $T_{c}=9.3 \mathrm{~K}$ for $\mathrm{Nb}_{1.9} \mathrm{Y}_{0.1} \mathrm{~B}_{2}$ and with $T_{c}=11.2 \mathrm{~K}$ for $\mathrm{Mo}_{1.69} \mathrm{Zr}_{0.31} \mathrm{~B}_{2}$.

The results of another systematic search for superconductivity in diborides were published by Leyarovska et al. [3]. Investigating magnetic susceptibility of $\mathrm{MeB}_{2}(\mathrm{Me}=\mathrm{Ti}, \mathrm{Zr}, \mathrm{Hf}, \mathrm{V}, \mathrm{Nb}, \mathrm{Ta}$, $\mathrm{Cr}, \mathrm{Mo}$ ) down to $0.42 \mathrm{~K}$ the authors found, that only $\mathrm{NbB}_{2}$ was superconducting with critical temperature equal to $T_{c}=0.62 \mathrm{~K}$. Among the materials studied there was not $\mathrm{MgB}_{2}$.
After discovery of Akimitsu [1] it was quite natural that many research groups rechecked old or newly prepared diborides. And the results obtained by different groups were quite surprising. Kaczorowski et al. [4] found superconducting signal in old $\mathrm{TaB}_{2}$ with $T_{c}=9.5 \mathrm{~K}$, but did not found it for $\mathrm{TiB}_{2}, \mathrm{HfB}_{2}, \mathrm{VB}_{2}, \mathrm{NbB}_{2}$ or $\mathrm{ZrB}_{2}$. In turn Gasparov et al. [5] found superconductivity in $\mathrm{ZrB}_{2}$ with $T_{c}=5.5 \mathrm{~K}$, but they did not confirm it existence for $\mathrm{TaB}_{2}$ and $\mathrm{NbB}_{2}$. Following the paper by Felner [6], that $\mathrm{BeB}_{2}$ is not superconducting, there was a paper by Young et al. [7] reporting superconductivity for $\mathrm{BeB}_{2.75}$ with $T_{c} \approx 0.7 \mathrm{~K}$. Superconductivity was also discovered [8] in $\mathrm{ReB}_{2}$ ( $T_{c}$ in the range 4.5-6.3 $\mathrm{K}$ depending on boron content) and $\operatorname{Re}_{3} \mathrm{~B}\left(T_{c}=4.7 \mathrm{~K}\right)$.

Substituting magnesium with monovalent, divalent or trivalent ions was studied by Medvedeva et al. [9]. Its influence on superconducting properties of $\mathrm{MgB}_{2}$ is connected with the electronic structure of the latter and dominated by the chemical bonding of the hexagonal, graphite-like B sheets. $\sigma$ bonds between the boron atoms are unfilled, so metallic, with more electrons in the $\pi$ bonds. In result $\mathrm{MgB}_{2}$ may be well characterized by the ionic form $\mathrm{Mg}^{2+}\left(\mathrm{B}_{2}\right)^{2-}$. Filling the holes in $\sigma$ band by trivalent substitution is unfavorable for superconductivity, while some monovalent substitutions may be in turn favorable for it. Such findings were partially supported as substitution of trivalent aluminum deteriorated superconductivity in the mate- 
rial [10] but monovalent copper substitution only broadened transition without changing the transition-onset value [11].

In our paper we describe the results of different method of doping of $\mathrm{MgB}_{2}$ - hydrogenation. We also mention about the results of doping $\mathrm{TaB}_{2}$ with hydrogen.

\section{Materials and methods}

In our investigations we made use of commercially available, powdered $\mathrm{MgB}_{2}$ (Johnson Matthey GmbH Alfa - 98\% purity). Hydrogen absorption was performed at a constant temperature under hydrogen gas pressure of $0.5-20$ bars. Our first attempts with reacting temperature about $250{ }^{\circ} \mathrm{C}$ resulted in decomposition of $\mathrm{MgB}_{2}$. So finally reaction temperature was established at $100{ }^{\circ} \mathrm{C}$ and pressure of 20 bars. Concentration of hydrogen was determined volumetrically by monitoring the pressure change in a calibrated, sealed volume. Under conditions described above hydrogen content was not higher than about $(3 \pm 1) \% /$ f.u. In attempt to increase the hydrogen uptake we have increased the hydrogen pressure up to $7 \mathrm{kbar}$, leaving temperature equal to $100{ }^{\circ} \mathrm{C}$. In this case the uptake of hydrogen was very similar, as measured by mass spectrometry method, and equal to about $3 \% /$ f.u. $\mathrm{X}$-ray analysis was carried out using STOE diffraction system. Material was characterized by the ac magnetic susceptibility measurements.

\section{Results and discussion}

$\mathrm{X}$-ray diffractograms for as obtained and hydratized samples is presented in Fig. 1. It is clearly seen that both the placement of diffraction peaks and their width is practically the same for all measured samples. Small changes of the peaks heights are to be ascribed, according to us, to the difference between the samples mass and thickness rather than to the different amounts of the majority phase. In principle such a result might be expected as the hydrogen uptake was rather small. Summing up - the structure and the volume of the phase with $\mathrm{MgB}_{2}$-type structure remained unchanged after hydrogenation.

According to the paper by Wan et al. [12] lattice constants values are connected with the placement of Fermi level comparing to the density of states (DOS). So the constancy of the lattice constants probably means that also DOS is not changing meaningfully.

The results of ac susceptibility measurements of $\mathrm{MgB}_{2}$ hydratized at 20 bar are presented in Fig. 2 .

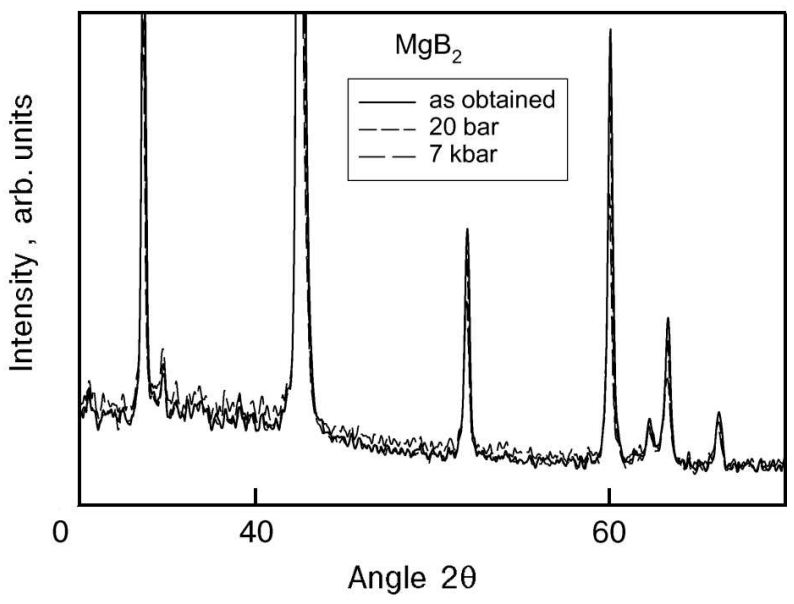

Fig. 1. X-ray diffractograms of as obtained $\mathrm{MgB}_{2}$ and hydrogenated under the pressure of $20 \mathrm{bar}$ and $7 \mathrm{kbar}$.

For comparison, also results for parent $\mathrm{MgB}_{2}$ are presented at the insets. The masses of both powdered samples were very similar. So the main features of the plots are:

- critical temperatures of both pure and hydratized compounds differs negligibly;

- superconducting response (so the amount of superconducting phase) is two orders of magnitude lower for hydratized sample;
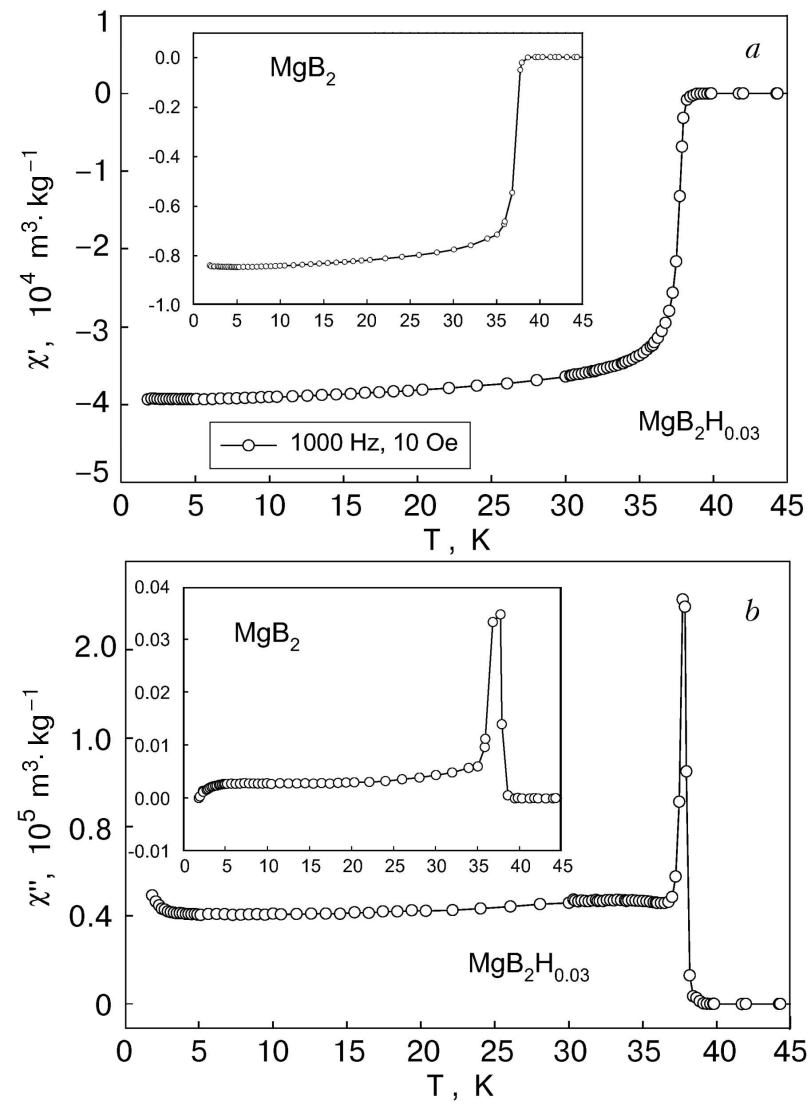

Fig. 2. Real (a) and imaginary (b) parts of ac susceptibility of $\mathrm{MgB}_{2}$ hydrogenated under pressure of $20 \mathrm{bar}$. Insets show the ac susceptibility for as obtained $\mathrm{MgB}_{2}$. 
- superconducting transition width is narrower for hydratized material.

At Fig. 3 the comparison between the ac susceptibilities of the $\mathrm{MgB}_{2}$ after hydrogenation under different hydrogen pressures is depicted. As it was mentioned above, the hydrogen uptake was very similar in both cases despite the fact that pressures during hydrogenation differs 350 times. And similarly to Fig. 2 we can observe that critical temperature remained unchanged, and that there is an additional decrease of the amount of superconducting phase.

Both facts - unchanged critical temperature and decreasing (with hydrogenation) amount of superconducting phase are rather surprising. First fact is implying that density of states, electron-phonon interaction and phonon spectrum remained unchanged after hydrogen uptake. In turn the decrease of the amount of superconducting phase may imply that density of states was meaningfully changed.

So to account for our observations, in the picture of conventional, BCS phonon mediated superconductor, the influence of hydrogenation on superconductivity should act in two opposing directions. We can analyze it using the modified McMillan equation [13]:

$$
k_{B} T_{c}=\frac{\hbar \omega_{0}}{1.2} \exp \left(-\frac{1.04(1+\lambda)}{\lambda-\mu^{*}(1+0.62 \lambda)}\right)
$$

where $k_{B}$ is Boltzman constant, $\omega_{0}$ is averaged phonon frequency, $\lambda$ is electron-phonon coupling constant, and $\mu^{*}$ is the Coulomb pseudopotential.

Hydrogenation can influence the phonon frequency. It was shown by Hinks et al. [14] that B atoms vibrations are more strongly coupled to the electronic structure than $\mathrm{Mg}$ ones. It may be inferred from the fact, that isotope effect for boron is much stronger than for magnesium one. Both theoretical calculation [15] and experimental work supports the view that $E_{2 g}$ in-plane boron stretching mode is the main source of strong electron-phonon coupling. This mode was found to be strongly anharmonic [16]. So if hydrogen is adsorbed within the boron planes (where it is easy to find the proper placement for it) it may strongly influence the stretching boron mode. But it is not easy to determine what kind of influence it can be. It might be increasing or lowering the anharmonicity or lowering or increasing phonon frequency. This way hydrogen can also change $\lambda$ or $\mu^{*}$.

Absorption of hydrogen within the Mg planes also can not be excluded. Such a placement should not change the important modes of phonon frequen-
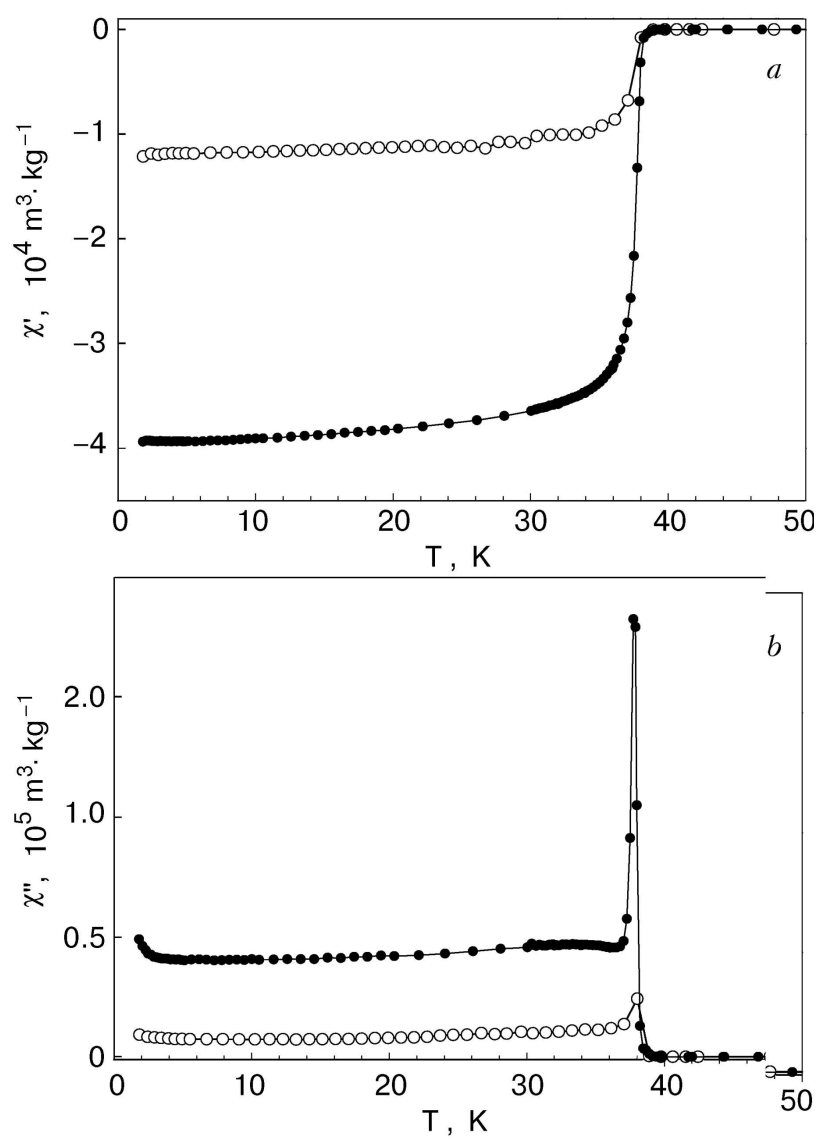

Fig. 3. The comparison of ac susceptibility measurements results for $\mathrm{MgB}_{2} \mathrm{H}_{0.03}$ obtained under hydrogen pressure $20 \mathrm{bar}$ (๑) and $7 \mathrm{kbar}(\bigcirc)$.

cies [14], but could influence the ionicity of the magnesium and boron planes [15]. This in turn should change the position of Fermi level with respect to the $\sigma$ boron bands and DOS. And with it also Coulomb screening and electron-phonon coupling could be changed. We believe, that the change of ionicity of the layers should influence the value of the lattice constant $c$, perpendicular to the planes. As it was not observed in our study, we are susceptible to consent to the first possibility.

It may be added that we apply the hydrogenation under low pressure and temperature to the controversial compound $\mathrm{TaB}_{2}$ studied by Kaczorowski et al. [4]. In this case hydrogen uptake was equal to $36 \% / \mathrm{f} . u$., much higher than for $\mathrm{MgB}_{2}$. And despite of such high hydrogen content, critical temperature remained unchanged and equal to about $9.5 \mathrm{~K}$, but diamagnetic signal was order of magnitude lower than for undoped material.

\section{Summary}

In conclusion we observed meaningful decrease of the diamagnetic signal of hydrogenated, commer- 
cial $\mathrm{MgB}_{2}$ powder, while its critical temperature remained practically unchanged. The hydrogen uptake was very low (about $4 \% /$ f.u.) and independent on the used pressure. Such strange behavior can be explained by some opposite influence of the hydrogen on DOS, phonon frequency, electron-phonon coupling and Coulomb screening.

The authors would like to express their gratitude for Dr. Marek Wolcyrz for x-ray measurements and Dr. Tadeusz Kopec for his valuable discussions.

1. J. Akimitsu, Symposium on Transition Metal Oxides, Sendai, January 10, 2001.

2. A. S. Cooper, E. Corenzwit, L. D. Longinotti, B. T. Matthias, and W. H. Zachariasen, Proc. Nat. Acad. Sci. 67, 313 (1970).

3. L. Leyarovska and E. Leyarovski, J. Less-Common Metals 67, 249 (1979).

4. D. Kaczorowski, A. J. Zaleski, O. J. Zogal, and J. Klamut, cond-mat/0103571.

5. V. A. Gasparov, N. S. Sidorov, I. Izver'kova, and M. P. Kulakov, cond-mat/0104323.
6. I. Felner, cond-mat/0102508.

7. D. P. Young, P. W. Adams, J. Y. Chan, and F. R. Fronczek, cond-mat/0104063.

8. G. K. Strukova, V. F. Degtyareva, D. V. Shovkun, V. N. Zverev, V. M. Kiiko, A. M. Ionov, and A. N. Chaika, cond-mat/0105293.

9. N. I. Medvedeva, A. I. Ivanovskii, J. E. Medvedeva, and A. J. Freeman, cond-mat/0103157.

10. J. S. Slusky, N. Rogado, K. A. Regan, M. A. Hayward, P. Khalifah, T. He, K. Inumaru, S. M. Loureiro, M. K. Haas, H. W. Zandbergen, and R. J. Cava, Nature 410, 343 (2001).

11. S. M. Kazakov, M. Angst, and J. Karpinski, condmat/0103350.

12. X. Wan, J. Dong, H. Weng, and D. Y. Xing, condmat/0104216.

13. W. L. McMillan, Phys. Rev. 167, 331 (1968).

14. D. G. Hinks, H. Claus, and J. D. Jorgensen, Nature 411, 457 (2001)

15. J. M. An and W. E. Pickett, cond-mat/0102391.

16. A. F. Goncharov, V. V. Struzhkin, E. Gregoryanz, J. Hu, R. J. Hemley, H.-K. Mao, G. Lapertot, S. L. Bud'ko, and P. C. Canfield, cond-mat $/ 0104042$. 